\title{
Elastic three-dimensional metaframe for selective wave filtering and polarization control
}

Cite as: Appl. Phys. Lett. 119, 211903 (2021); https://doi.org/10.1063/5.0065553

Submitted: 03 August 2021 • Accepted: 10 November 2021 • Published Online: 23 November 2021

(D) J. M. De Ponti, (D) E. Riva, (D) F. Braghin, et al.
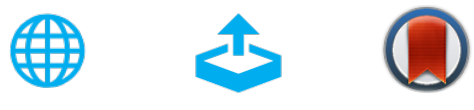

View Online

Export Citation

\section{ARTICLES YOU MAY BE INTERESTED IN}

Actively tunable photonic crystal-based switch via plasmon-analog of index enhancement Applied Physics Letters 119, 211103 (2021); https://doi.org/10.1063/5.0071984

Polarization-controlled generation and superposition of surface plasmon polariton vortices with a plasmonic metasurface

Applied Physics Letters 119, 211102 (2021); https://doi.org/10.1063/5.0070339

One-dimensional covalent organic framework-Carbon nanotube heterostructures for efficient capacitive energy storage

Applied Physics Letters 119, 211905 (2021); https://doi.org/10.1063/5.0073426

\section{Challenge us.}

What are your needs for periodic signal detection?

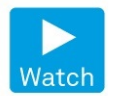

- Zurich

Instruments

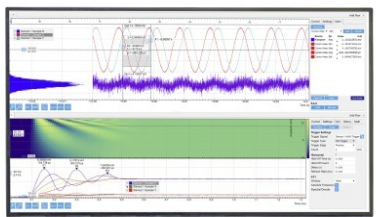

(- 


\title{
Elastic three-dimensional metaframe for selective wave filtering and polarization control
}

\author{
Cite as: Appl. Phys. Lett. 119, 211903 (2021); doi: 10.1063/5.0065553 \\ Submitted: 3 August 2021 - Accepted: 10 November 2021 . \\ Published Online: 23 November 2021
}

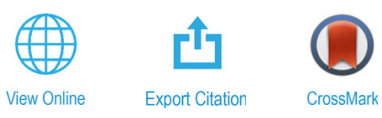

\section{J. M. De Ponti, ${ }^{1, a)}$ (D) E. Riva, ${ }^{2}$ (D) F. Braghin, ${ }^{2}$ (iD and R. Ardito ${ }^{1}$ (iD}

\begin{abstract}
AFFILIATIONS
${ }^{7}$ Department of Civil and Environmental Engineering, Politecnico di Milano, Piazza Leonardo da Vinci 32, 20133 Milano, Italy

${ }^{2}$ Department of Mechanical Engineering, Politecnico di Milano, Via Giuseppe La Masa 1, 20156 Milano, Italy
\end{abstract}

${ }^{\text {a) }}$ Author to whom correspondence should be addressed: jacopomaria.deponti@polimi.it

\begin{abstract}
We experimentally achieve selective wave filtering and polarization control in a three-dimensional elastic frame embedding local resonators. By connecting multi-resonating elements to a frame structure, a complete low-frequency, subwavelength bandgap with strong selective filtering properties is obtained. Theory and experiments demonstrate the metaframe capability to selectively stop transverse waves while allowing longitudinal wave propagation as in "fluid-like" elasticity. This peculiar behavior, together with the complete bandgap structure, may open opportunities in the context of wave control, envisaging concurrent applications for three-dimensional filters and elastic wave polarizers.
\end{abstract}

Published under an exclusive license by AIP Publishing. https://doi.org/10.1063/5.0065553

A dominant line of research in wave physics focuses on the investigation of materials and structures to control the propagation of waves. Periodic media have been particularly influential to manipulate waves exploiting Bragg scattering bandgaps, creating the fields of photonic crystals in optics ${ }^{1}$ and their acoustic counterparts in phononic structures. $^{2-4}$ In the context of elastic waves, notable effort has been devoted to the definition of low frequency and subwavelength devices to comply with ambient and most widespread vibration spectra. To push the operational regime toward lower frequencies, the exploitation of local resonance has received considerable attention, ${ }^{5-8}$ leading to the definition of metamaterials. ${ }^{9,10}$ Potential applications of elastic metamaterials could be implemented at any length scale, for seismic wave protection, ${ }^{11-16}$ vibration absorption, ${ }^{17-20}$ nondestructive evaluation, ${ }^{21}$ and several nuances for wave enhancement and manipulation $^{22-24}$ recently inspired by topological insulators. ${ }^{25-27}$ Metamaterials are often employed in combination with smart materials to obtain multifunctional devices for enhanced sensing or energy harvesting applications. ${ }^{28-30}$ The effect of local resonance on the dispersion properties in continuous elastic substrates has been widely investigated in the past through analytical and approximated formulations in beams, ${ }^{31,32}$ plates, ${ }^{33-35}$ and halfspaces, ${ }^{35,36}$ among others. Motivated by the idea to control elastic waves in a complete threedimensional setting, we propose a metaframe able to provide selective wave filtering and polarization control. Moreover, we show that simple lumped models can be suitably employed to predict the behavior of such structures, providing a rapid analytical design tool. We show that the attenuation performance of locally resonant structures strongly depends on the relative stiffness ratio (frame vs resonator), which should be considered an additional parameter to the conventional total mass ratio. ${ }^{32}$ Even if a complete bandgap is obtained, the imaginary dispersion curves are remarkably different for longitudinal and transverse waves; this behavior is linked to the stiffness ratio between the frame and the resonator, which affects the attenuation properties of the structure. More specifically, we demonstrate selective attenuation of transverse waves, while allowing longitudinal wave propagation as in fluidlike elasticity. ${ }^{37,38}$ The possibility to create polarization bandgaps by a selective suppression of each vibration mode has been demonstrated in stiff-in-soft structures with a single resonator per cell, obtaining different bandgaps for flexural, longitudinal, or torsional modes ${ }^{37}$ or leveraging multiple resonances. ${ }^{38}$ Modes can also be isolated on the basis of their polarization by creating distinct topological interfaces ${ }^{39}$ supporting heterogeneous helical-valley edge waves. Differently from these approaches, we show a three-dimensional metaframe with the coexistence of attenuation and transmission of transverse and longitudinal waves, both inside a common bandgap.

Although this mechanism may resemble an elastic threedimensional analogue of optical waveguide polarizers in photonics, ${ }^{40,41}$ the concept of wavelength selective filtering is caused here by a complete bandgap with an imaginary component strongly dependent on the wave polarization. This can be regarded as an additional feature coming from the higher complexity of wave polarization in elasticity, which allows creating complete bandgaps with strong selective filtering properties. This mechanism can be employed for the creation of threedimensional single phase selective filters or elastic wave polarizers. 
On the basis of previous research results, ${ }^{42}$ we consider the metaframe depicted in Fig. 1, obtained connecting local resonators to an elastic frame. The finite structure is defined by periodically repeating a unit cell containing eight resonators made of a thin elastic beam equipped with a sphere on the tip and connected to a frame node. Such arrangement allows one to increase the resonating mass, while keeping the stiffness of the resonators low.

The unit cell, of size $a=50 \mathrm{~mm}$, includes a frame with beams of a square cross section of dimension $w_{\text {frame }}=0.08 a$, while the resonators are characterized by a cylindrical elastic connection of diameter $w_{\text {res }}=0.04 a$, length $l_{\text {res }}=0.14 a$ and a sphere of radius $r_{\text {sphere }}=0.21 a$. The entire structure is made of Nylon PA12 with Young's modulus $E_{P A 12}=1586 \mathrm{MPa}$, Poisson's ratio $\nu=0.4$, and density $\rho=1000 \mathrm{~kg} / \mathrm{m}^{3}$.

The numerical dispersion curves of the metaframe, obtained through Abaqus ${ }^{\circledR}$, are displayed in Fig. 2(a), along with the graphical representation of a number of Bloch modes. A complete lowfrequency bandgap in the range of $58.04-83.28 \mathrm{~Hz}$ with a gap-midgap ratio ${ }^{42}$ of $35.2 \%$ is observed. In terms of the normalized frequency, i.e., in units of the resonance frequency, we obtain a bandgap in the range 0.97-1.39. In correspondence of the bandgap edges, the Bloch modes exhibit a localized flexural motion of the resonators with in-phase and out-of-phase response for the mode below and above, respectively. At this step, we also observe that the number of propagating wave modes that collapse in a bandgap can be dynamically affected in a different manner from the resonator motion due to the associated equivalent stiffness, which is very different for longitudinal and transverse waves. Figure 2(b) shows the wave propagation along an infinitely long elastic frame with the insertion of the metaframe composed of nine unit cells. Spurious edge reflections are avoided by imposing absorbing boundary conditions at the end of the frame along the wave propagation direction. They are implemented in Abaqus using the ALID (absorbing layers using increasing damping) method, adopting a cubic law function for mass proportional Rayleigh damping. ${ }^{43}$ By inspecting the

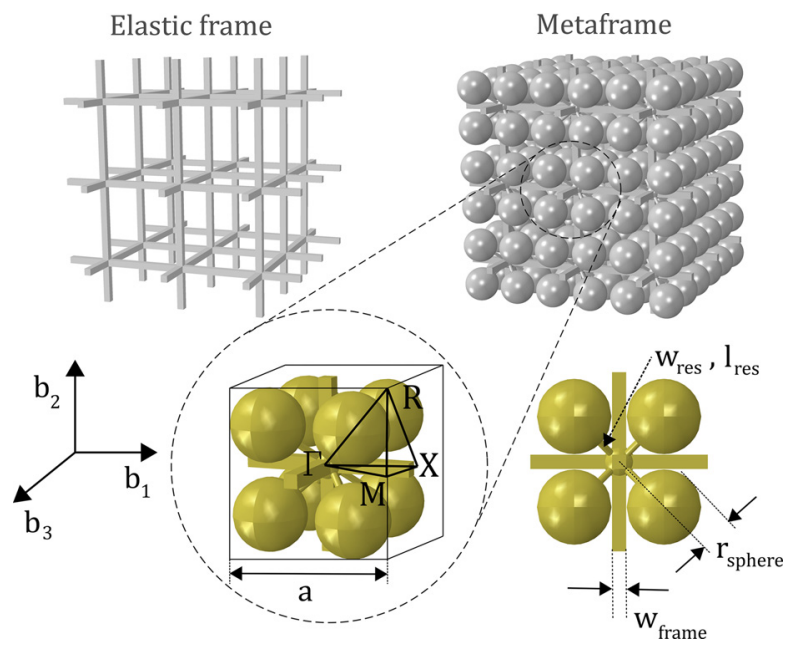

FIG. 1. Elastic three-dimensional metaframe. The structure is obtained by connecting a set of resonators to the nodes of an elastic frame. In the bottom part, a detailed view of the unit cell made of a frame with eight resonators attached is shown, together with the main geometrical dimensions and the high symmetry points used to compute the dispersion curves. wave amplitude after the metaframe, it can be noticed that, for a constant input excitation, longitudinal waves propagate, while transverse waves are attenuated. This phenomenon is observed independently on the metaframe dimension, as can be noticed considering larger samples (see the supplementary material). While the influence of different resonator modes on the attenuation performances has already been investigated, ${ }^{33,35}$ comparing for, e.g., flexural and longitudinal resonances, we focus our attention on a single mode of the resonator, changing the stiffness of the frame only. This is possible thanks to the three-dimensional symmetry of the cell, which guarantees a flexural polarization of the resonators in accord with both longitudinal and transverse waves.

To investigate on this aspect, we adopt a simplified lumped model to predict the bandgap characteristics and the associated level of attenuation. We consider a periodic spring-mass chain, made of unit cells of stiffness $k$ and mass $M$, with attached a resonator of stiffness $k_{R}$ and mass $m_{R}$, as shown in the inset of Fig. 3. The quantity $k$ represents the stiffness of the plain frame, while $m$ is the overall nonresonating mass. We adopt two degrees of freedom to describe the translation of the main mass $M$ and of the resonator $m_{R}$.

By defining $\Omega^{2}=k / M, \omega_{R}^{2}=k_{R} / m_{R}$ and introducing the nondimensional quantity $\mu=\kappa d$, being $d$ the unit cell size and $\kappa$ the wavenumber, the dispersion relation is

$$
\cos (\mu)=1-\frac{\omega^{2}}{2 \Omega^{2}}\left[1+\frac{m_{R}}{M} \frac{\omega_{R}^{2}}{\omega_{R}^{2}-\omega^{2}}\right] .
$$

Evanescent waves are present if $\cos (\mu) \geq 1$ and $\cos (\mu) \leq-1$. By imposing these conditions, we define, after some algebraic manipulations (see the supplementary material), the limits of the bandgap as

$$
\frac{4 \Omega^{2}+\omega_{R}^{2}\left(1+\frac{m_{R}}{M}\right)-\sqrt{\left[4 \Omega^{2}+\omega_{R}^{2}\left(1+\frac{m_{R}}{M}\right)\right]^{2}-16 \omega_{R}^{2} \Omega^{2}}}{2}
$$

$$
\begin{gathered}
\leq \omega^{2} \leq \omega_{R}^{2}\left(1+\frac{m_{R}}{M}\right) \\
\omega^{2} \geq \frac{4 \Omega^{2}+\omega_{R}^{2}\left(1+\frac{m_{R}}{M}\right)+\sqrt{\left[4 \Omega^{2}+\omega_{R}^{2}\left(1+\frac{m_{R}}{M}\right)\right]^{2}-16 \omega_{R}^{2} \Omega^{2}}}{2} .
\end{gathered}
$$

The equations reveal that the bandgap does not open at the resonance frequency $\omega_{R}$ but at a frequency that depends also on the inertial and elastic properties of the main structure.

The longitudinal (axial) and transverse (bending) stiffness of the frame can be easily computed by means of well-known techniques of structural mechanics (see the supplementary material). Moreover, we model the resonator as a Timoshenko elastic beam with a lumped rigid mass, assuming as Lagrangian coordinates the translation of the tip of the beam and the rotation of the mass. By doing so, we also neglect the mass of the beam $(0.46 \%$ of the one of the spheres) and assume an infinite longitudinal (axial) stiffness. By solving the eigenvalue problem for the two degrees of the freedom model of the resonator, we obtain that $82.4 \%$ of the mass of the sphere participates in the motion at the resonance frequency $f_{R}=60.7 \mathrm{~Hz}$. Having computed the mass and stiffness parameters, we get from Eq. (1) the analytical dispersion 

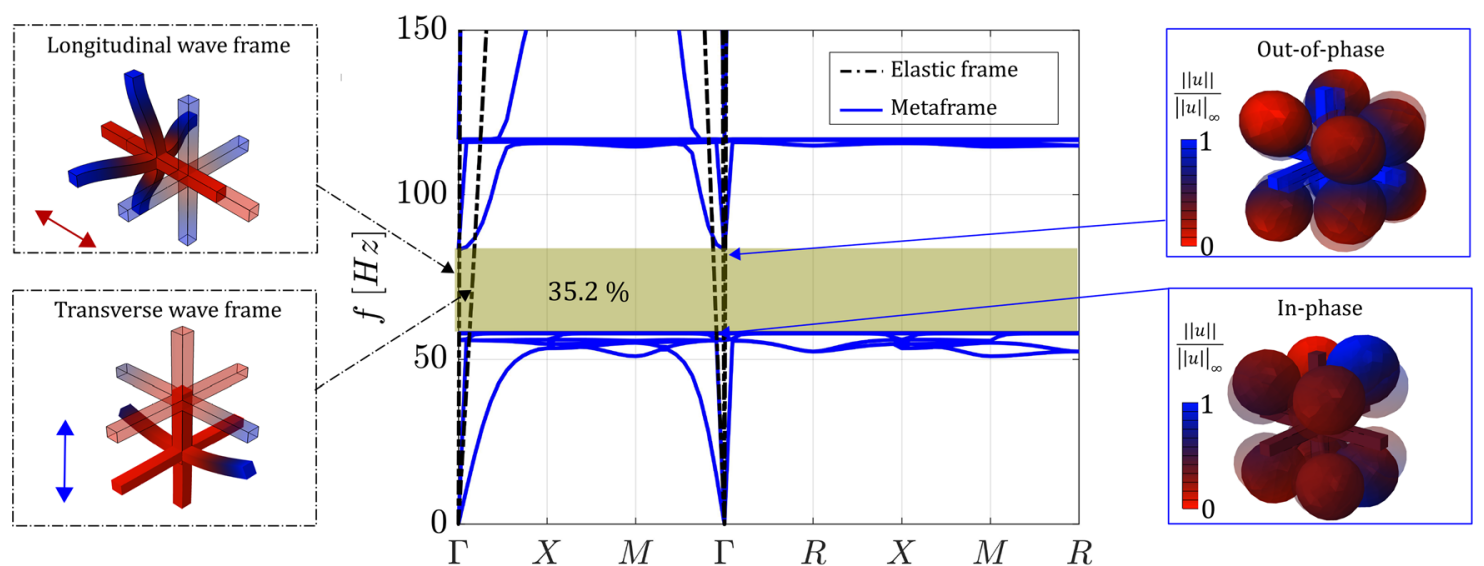

(a)
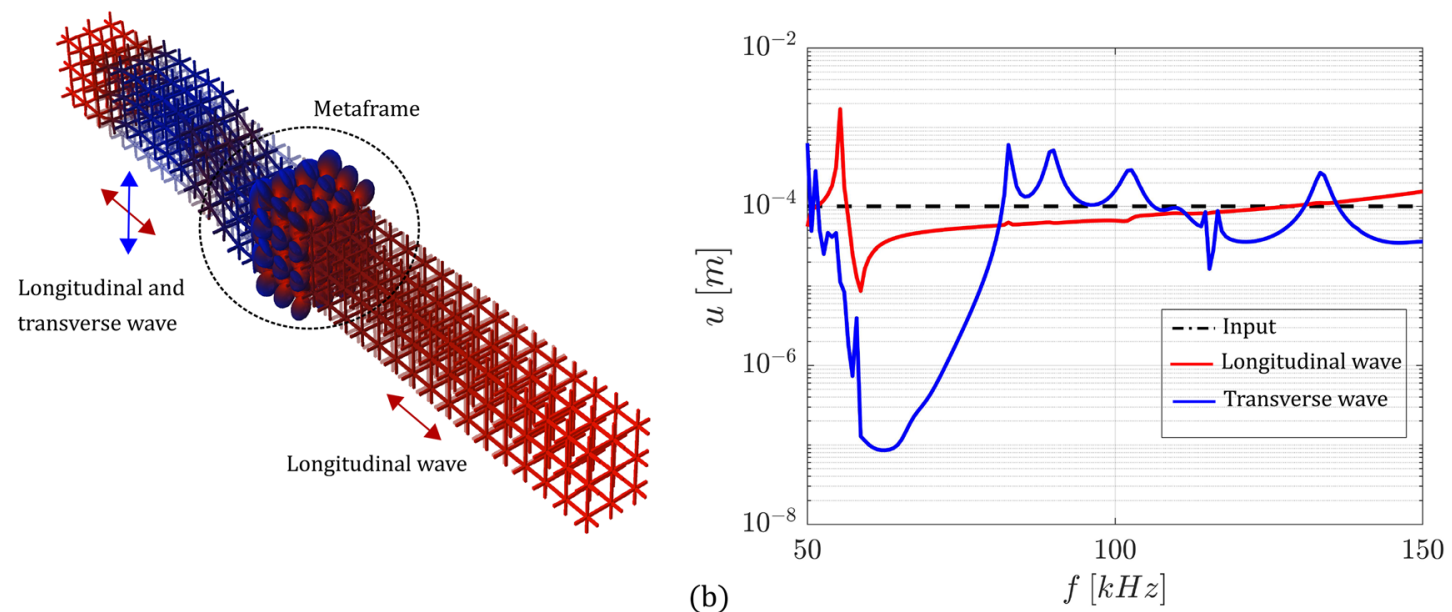

FIG. 2. (a) Numerical dispersion curves of the metaframe obtained through FEM, superimposed to the dispersion of a plain elastic frame without the resonators. A low frequency bandgap with a gap-midgap ratio of $35.2 \%$ is observed in the range $58.04-83.28 \mathrm{~Hz}$ for both longitudinal and transverse waves. Insets show the vibration modes of the plain frame and the metaframe at the $\Gamma$ point of the bandgap edges. The bandgap opens with an in-phase response of the resonators, while the closure is related to outof-phase oscillations. (b) Wave propagation analyses on an infinitely long elastic frame embedding the metaframe. Selective wave filtering is observed: for the same input, longitudinal waves propagate, while transverse waves are attenuated.

curve reported in Fig. 3. A good agreement between the lumped and the three-dimensional finite element models is observed. The simplified model provides accurate predictions in the frequency range of interest with an error of approximately $4 \%$ for the bandgap opening and closing frequencies. The plot shows clearly that the bandgap opening frequency for the transverse excitation is significantly smaller than the case of the longitudinal wave. As a consequence, there is a frequency range in which transverse waves are evanescent and longitudinal waves are amplified; the relative width of such a range, with respect to the midrange frequency, is equal to $13 \%$. More importantly, the lumped model is used to easily evaluate the imaginary part of the dispersion relation, which quantifies the actual level of attenuation provided within the bandgap. ${ }^{3}$ It can be noticed that transverse waves are strongly attenuated, while the longitudinal ones are slightly affected by the bandgap, except for a narrow region centered at the resonance frequency of the resonator. This feature allows the metaframe to selectively attenuate transverse waves and enable longitudinal wave propagation. That is, for a mixed longitudinal and transverse polarization of the input at the bandgap frequencies, one can make a filter for transverse wave components, while keeping unaltered the longitudinally polarized waves. The different attenuation levels are attributed to the stronger mismatch between the axial stiffness of the frame and the flexural stiffness of the resonators. In terms of waves, a higher wavelength is obtained, thus increasing the subwavelength requirements of the system.

The theoretical results are hereafter validated experimentally. Prototypes are built using selective laser sintering (SLS), an additive manufacturing process that has no need of support material (a critical issue for the realization of suspended parts, like the resonators in this case) while guaranteeing good precision. The finite structure is composed of nine unit cells, resulting in a cube of dimension $150 \mathrm{~mm}$. The experimental setup is shown in Fig. 4.

The metaframe is glued to an aluminum thick plate, which is connected to a LDS v406 electrodynamic shaker to provide excitation. 


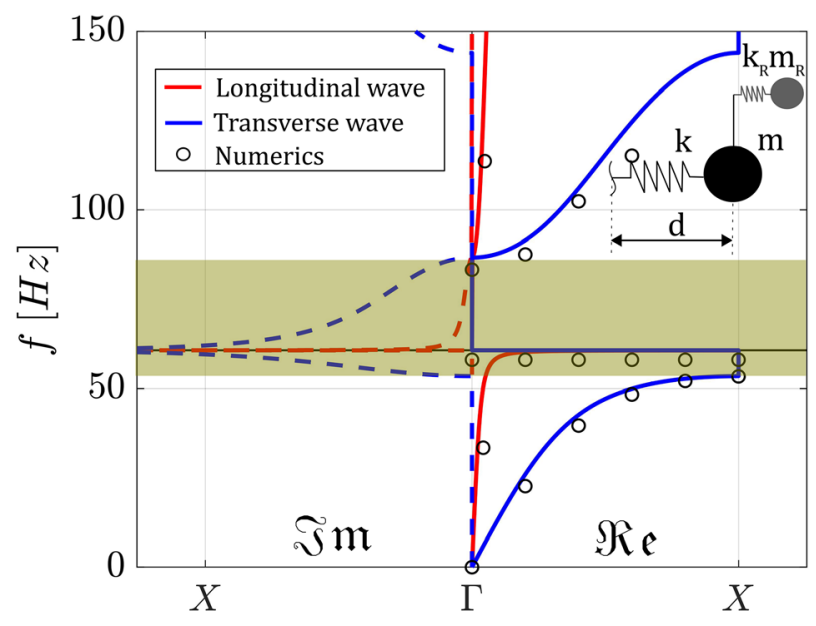

FIG. 3. Analytical dispersion curve for the metaframe, superimposed to the numerical dispersion (scattered points) from FEM. Even if the bandgap exists for both longitudinal and transverse waves, the imaginary part of the dispersion curves is remarkably different, reporting almost negligible attenuation for longitudinal waves.

The aluminum plate is sufficiently thick to avoid relevant deformations for the input frequency, i.e., it is assumed as a perfectly rigid connection between the metaframe and the shaker. Two configurations are tested, imposing a longitudinal and a transverse polarization of the wave, respectively. Both cases consider the same direction of the wave propagation, $b_{1}$ in Fig. 1 , but different polarization, i.e., $b_{1}$ for longitudinal waves and $b_{3}$ for transverse waves. For the longitudinal wave, the plate is directly mounted onto the shaker, which provides a vertical excitation. In contrast, the transverse wave is obtained by rotating the

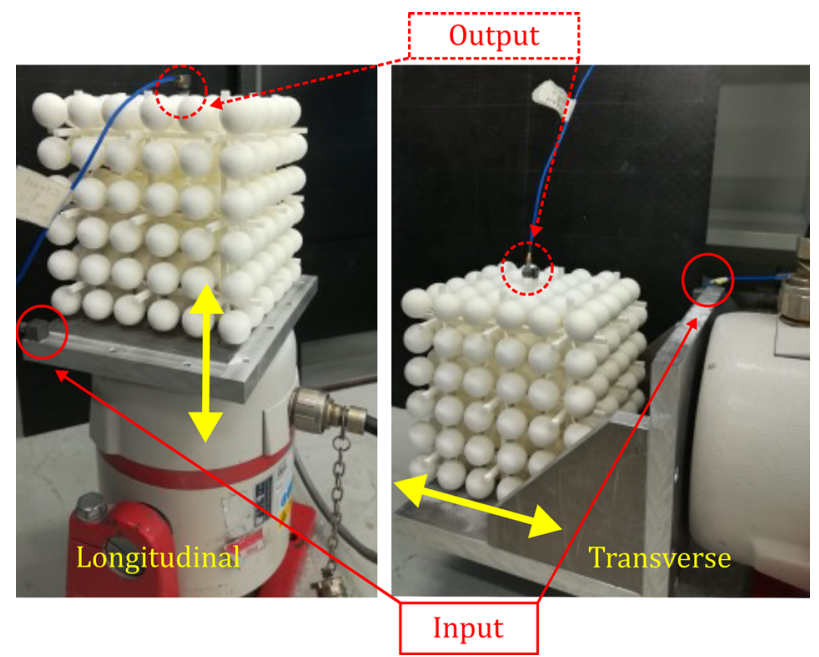

FIG. 4. Experimental setup. The excitation is provided through an electrodynamic shaker. The metaframe is glued to an aluminum thick plate, which is rigidly connected to the shaker. Longitudinal excitation is provided by vertically placing the plate onto the shaker. For transverse excitation, the shaker is rotated of $90^{\circ}$ and the prototype is placed on a $L$-shaped fixture. Input and output accelerations are measured through accelerometers glued on the rigid plate and on the metaframe, respectively. shaker of $90^{\circ}$ and connecting the plate to a $L$-shaped structure. The experimental acceleration spectra are measured using PCB Piezotronics 352C33 accelerometers with a sensitivity of $10 \mathrm{mV} / \mathrm{g}$ and the resonant frequency higher than $70 \mathrm{kHz}$. Since the accelerometers are able to measure the acceleration along one direction only, we rotated them of $90^{\circ}$ to measure transverse waves. The input is measured through an accelerometer glued to the thick plate, while the output is measured on the frame structure at the opposite side, as indicated by red circles and arrows in Fig. 4. The excitation signal is provided through a KEYSIGHT 33500B waveform generator, which synchronously starts with the measurement system and consists in a linear swept-frequency cosine signal modulated by a Hann window. A frequency range between 50 and $150 \mathrm{~Hz}$ is considered, filtering out the other frequency components. Data are then post-processed in order to estimate the transmission spectra, defined as $\tau ;(\mathrm{dB})=20$ $\log _{10}\left(\ddot{w}_{\text {OUT }} / \ddot{w}_{I N}\right)$.

Figure 5 shows the numerical and experimental transmission diagrams for longitudinal and transverse waves. To investigate the results, we carry out FEM viscoelastic transmission analyses ${ }^{44,45}$ using the standard linear solid-Maxwell type-model with $\tau_{\text {Maxwell }}=1.5 \times 10^{-4} \mathrm{~s}$ and Young's modulus $E_{\text {Maxwell }}=315 \mathrm{MPa}$. The experimental data are in satisfactory agreement with the computations, corroborating the different attenuation performance of the structure for longitudinal and transverse waves. The different level of attenuation and the slight shift in the position of the transmission peaks are attributed to the relative simplicity of the viscoelastic model with respect to the actual material behavior, as well as experimental misalignments that prevent the possibility to excite longitudinal and transverse waves separately.

In conclusion, we have experimentally demonstrated potential advantages in using a three-dimensional metaframe for the creation of a complete, low-frequency, subwavelength bandgap. The strong mismatch between the longitudinal and transverse stiffness of the frame allows one to achieve a different control of the waves depending on

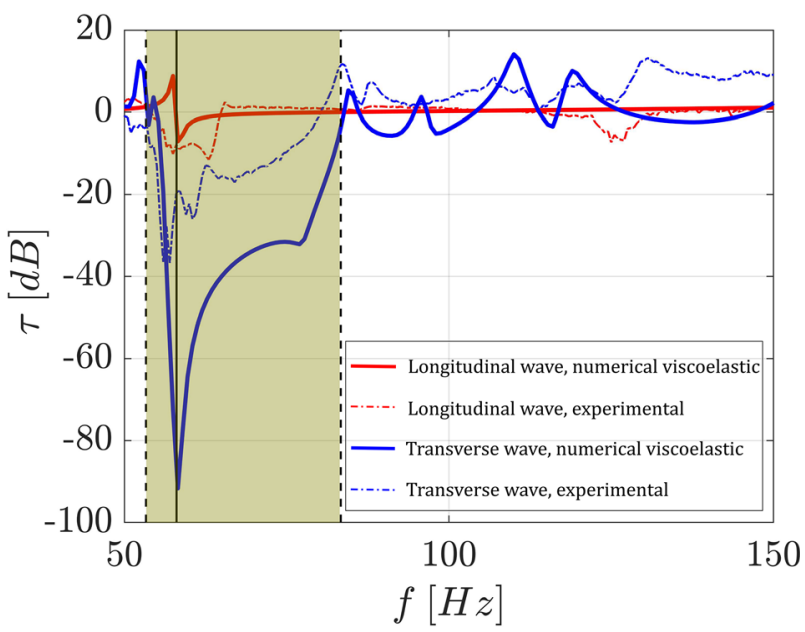

FIG. 5. Numerical FEM viscoelastic (continuous lines) and experimental (dasheddot lines) transmission spectra for longitudinal and transverse waves. Longitudinal waves (red lines) are slightly attenuated inside the expected bandgap region; maximum attenuation is detected at the resonance frequency of the resonators, marked by the continuous black vertical line. A stronger attenuation is measured for transverse waves (blue lines) in agreement with previous dispersion curves predictions. 
the polarization direction. Strong attenuation of transverse waves is achieved, while longitudinal wave propagation is mostly unaffected by the bandgap, except from a sharp region close to the resonant frequency of the resonators. This behavior can be suitably employed for applications involving selective wave filtering and polarization control.

See the supplementary material for a detailed description of the analytical and numerical models.

The support of the H2020 FET-proactive project MetaVEH under Grant Agreement No. 952039 is acknowledged. We also gratefully acknowledge the Italian Ministry of Education, University and Research for the support provided through the Project "Department of Excellence LIS4.0-Lightweight and Smart Structures for Industry 4.0."

\section{AUTHOR DECLARATIONS \\ Conflict of Interest}

The authors have no conflicts to disclose.

\section{DATA AVAILABILITY}

The data that support the findings of this study are available from the corresponding author upon reasonable request.

\section{REFERENCES}

${ }^{1}$ J. D. Joannopoulos, S. G. Johnson, J. N. Winn, and R. D. Meade, Photonic Crystals, Molding the Flow of Light, 2nd ed. (Princeton University Press, Princeton, NJ, 2008).

${ }^{2}$ M. S. Kushwaha, P. Halevi, L. Dobrzynski, and B. Djafari-Rouhani, “Acoustic band structure of periodic elastic composites,” Phys. Rev. Lett. 71, 2022 (1993).

${ }^{3}$ V. Laude, Phononic Crystals, Artificial Crystals for Sonic, Acoustic, and Elastic Waves, De Gruyter Studies in Mathematical Physics (De Gruyter, 2015), Vol. 26.

${ }^{4}$ M. I. Hussein, M. J. Leamy, and M. Ruzzene, "Dynamics of phononic materials and structures: Historical origins, recent progress, and future outlook," Appl, Mech. Rev. 66(4), 040802 (2014)

${ }^{5}$ Z. Liu, X. Zhang, Y. Mao, Y. Y. Zhu, Z. Yang, C. T. Chan, and P. Sheng, "Locally resonant sonic materials," Science 289(5485), 1734-1736 (2000).

${ }^{6} \mathrm{G}$. Ma and P. Sheng, "Acoustic metamaterials: From local resonances to broad horizons," Sci. Adv. 2(2), e1501595 (2016).

${ }^{7}$ A. E. Miroshnichenko, S. Flach, and Y. S. Kivshar, "Fano resonances in nanoscale structures,” Rev. Mod. Phys. 82, 2257-2298 (2010).

${ }^{8}$ F. Lemoult, M. Fink, and G. Lerosey, "Acoustic resonators for far-field control of sound on a subwavelength scale,” Phys. Rev. Lett. 107, 064301 (2011).

${ }^{9}$ R. V. Craster and S. Guenneau, Acoustic Metamaterials Negative Refraction, Imaging, Lensing and Cloaking, Springer Series in Materials Science (Springer, 2013).

${ }^{10}$ R. V. Craster and S. Guenneau, World Scientific Handbook of Metamaterials and Plasmonics: Volume 2: Elastic, Acoustic and Seismic Metamaterials (World Scientific, Singapore, 2017).

${ }^{11}$ S. Brûlé, E. H. Javelaud, S. Enoch, and S. Guenneau, "Experiments on seismic metamaterials: Molding surface waves,” Phys. Rev. Lett. 112, 133901 (2014).

${ }^{12}$ Y. Achaoui, T. Antonakakis, S. Brule, R. V. Craster, S. Enoch, and S. Guenneau, "Clamped seismic metamaterials: Ultra-low frequency stop bands," New J. Phys. 19, 063022 (2017)

${ }^{13}$ G. Finocchio, O. Casablanca, G. Ricciardi, U. Alibrandi, F. Garescì, M. Chiappini, and B. Azzerboni, "Seismic metamaterials based on isochronous mechanical oscillators," Appl. Phys. Lett. 104, 191903 (2014).

${ }^{14}$ A. Colombi, P. Roux, S. Guenneau, P. Gueguen, and R. V. Craster, "Forests as a natural seismic metamaterial: Rayleigh wave bandgaps induced by local resonances," Sci. Rep. 6, 19238 (2016).
${ }^{15}$ M. Miniaci, A. Krushynska, F. Bosia, and N. M. Pugno, "Large scale mechanical metamaterials as seismic shields," New J. Phys. 18, 083041 (2016).

${ }^{16}$ A. Colombi, D. Colquitt, P. Roux, S. Guenneau, and R. V. Craster, "A seismic metamaterial: The resonant metawedge,” Sci. Rep. 6, 27717 (2016).

${ }^{17}$ A. Colombi, V. Ageeva, R. J. Smith, A. Clare, R. Patel, M. Clark, D. Colquitt, P. Roux, S. Guenneau, and R. V. Craster, "Enhanced sensing and conversion of ultrasonic Rayleigh waves by elastic metasurfaces,” Sci. Rep. 7, 6750 (2017).

${ }^{18}$ K. H. Matlack, A. Bauhofer, S. Krödel, A. Palermo, and C. Daraio, "Composite 3D-printed metastructures for low-frequency and broadband vibration absorption,” Proc. Natl. Acad. Sci. 113, 8386-8390 (2016).

${ }^{19}$ N. Kaina, M. Fink, and G. Lerosey, "Composite media mixing Bragg and local resonances for highly attenuating and broad bandgaps," Sci. Rep. 3, 3240 (2013).

${ }^{20} \mathrm{M}$. Moscatelli, R. Ardito, L. Driemeier, and C. Comi, "Band-gap structure in two- and three-dimensional cellular locally resonant materials," J. Sound Vib. 454, 73-84 (2019).

${ }^{21} \mathrm{M}$. Molerón and C. Daraio, "Acoustic metamaterial for subwavelength edge detection,” Nat. Commun. 6, 8037 (2015).

${ }^{22}$ V. Romero-García, R. Picó, A. Cebrecos, V. J. Sánchez-Morcillo, and K. Staliunas, "Enhancement of sound in chirped sonic crystals," Appl. Phys. Lett. 102, 091906 (2013)

${ }^{23}$ Y. Chen, H. Liu, M. Reilly, H. Bae, and M. Yu, "Enhanced acoustic sensing through wave compression and pressure amplification in anisotropic metamaterials," Nat. Commun. 5, 5247 (2014).

${ }^{24}$ Y. Liu, Z. Liang, F. Liu, O. Diba, A. Lamb, and J. Li, "Source illusion devices for flexural lamb waves using elastic metasurfaces," Phys. Rev. Lett. 119, 034301 (2017).

${ }^{25}$ S. H. Mousavi, A. B. Khanikaev, and Z. Wang, "Topologically protected elastic waves in phononic metamaterials," Nat. Commun. 6, 8682 (2015).

${ }^{26}$ R. Süsstrunk and S. D. Huber, "Observation of phononic helical edge states in a mechanical topological insulator," Science 349(6243), 47-50 (2015).

${ }^{27}$ G. J. Chaplain, J. M. De Ponti, G. Aguzzi, A. Colombi, and R. V. Craster, "Topological rainbow trapping for elastic energy harvesting in graded SuSchrieffer-Heeger systems," Phys. Rev. Appl. 14, 054035 (2020).

${ }^{28} \mathrm{C}$. Sugino and A. Erturk, "Analysis of multifunctional piezoelectric metastructures for low-frequency bandgap formation and energy harvesting," J. Phys. D 51, 215103 (2018).

${ }^{29}$ J. M. De Ponti, A. Colombi, R. Ardito, F. Braghin, A. Corigliano, and R. V. Craster, "Graded elastic metasurface for enhanced energy harvesting," New J. Phys. 22, 013013 (2020).

${ }^{30}$ J. M. De Ponti, A. Colombi, E. Riva, R. Ardito, F. Braghin, A. Corigliano, and R. V. Craster, "Experimental investigation of amplification, via a mechanical delay-line, in a rainbow-based metamaterial for energy harvesting," Appl. Phys. Lett. 117, 143902 (2020).

${ }^{31} \mathrm{E}$. Baravelli and M. Ruzzene, "Internally resonating lattices for bandgap generation and low-frequency vibration control," J. Sound Vib. 332(25), 6562-6579 (2013).

${ }^{32}$ C. Sugino, S. Leadenham, M. Ruzzene, and A. Erturk, "On the mechanism of bandgap formation in locally resonant finite elastic metamaterials," J. Appl. Phys. 120, 134501 (2016)

${ }^{33}$ E. G. Williams, P. Roux, M. Rupin, and W. A. Kuperman, “Theory of multire-

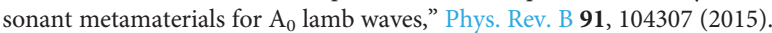

${ }^{34} \mathrm{~J}$. Y. Yoritomo and R. L. Weaver, "On band gap predictions for multiresonant metamaterials on plates,” J. Acoust. Soc. Am. 139, 1282 (2016).

${ }^{35}$ D. J. Colquitt, A. Colombi, R. V. Craster, P. Roux, and S. R. L. Guenneau, "Seismic metasurfaces: Sub-wavelength resonators and Rayleigh wave interaction," J. Mech. Phys. Solids 99, 379-393 (2017).

${ }^{36}$ C. Comi, M. Moscatelli, and J. J. Marigo, "Two scale homogenization in ternary locally resonant metamaterials," Mater. Phys. Mech. 44(1), 8-18 (2020).

${ }^{37}$ G. Ma, C. Fu, G. Wang, P. del Hougne, J. Christensen, Y. Lai, and P. Sheng, "Polarization bandgaps and fluid-like elasticity in fully solid elastic metamaterials," Nat. Commun. 7, 13536 (2016).

${ }^{38}$ Y. Lai, Y. Wu, P. Sheng, and Z.-Q. Zhang, "Hybrid elastic solids," Nat. Mater. 10, 620-624 (2011).

${ }^{39}$ M. Miniaci, R. K. Pal, R. Manna, and M. Ruzzene, "Valley-based splitting of topologically protected helical waves in elastic plates," Phys. Rev. B 100, 024304 (2019). 
${ }^{40} \mathrm{R}$. K. Sinha and Y. Kalra, "Design of optical waveguide polarizer using photonic band gap,” Opt. Express 14(22), 10790-10794 (2006).

${ }^{41}$ J. Zimmermann, M. Kamp, A. Forchel, and R. März, "Photonic crystal waveguide directional couplers as wavelength selective optical filters," Opt. Commun. 230(4-6), 387-392 (2004).

${ }^{42}$ L. D'Alessandro, E. Belloni, G. D'Alo, L. Daniel, R. Ardito, A. Corigliano, and F. Braghin, "Modelling and experimental verification of a single phase threedimensional lightweight locally resonant elastic metamaterial with complete low frequency bandgap," in 11th International Congress on Engineered Material Platforms for Novel Wave Phenomena, Metamaterials (IEEE, 2017), pp. 70-72.
${ }^{43}$ P. Rajagopal, M. Drozdz, E. A. Skelton, M. J. S. Lowe, and R. V. Craster, "On the use of absorbing layers to simulate the propagation of elastic waves in unbounded isotropic media using commercially available finite element packages," NDT\&E Int. 51, 30-40 (2012).

${ }^{44}$ A. Krushynska, V. G. Kouznetsova, and M. G. D. Geers, "Visco-elastic effects on wave dispersion in three-phase acoustic metamaterials," J. Mech. Phys. Solids 96, 29-47 (2016).

${ }^{45}$ L. D’Alessandro, A. Krushynska, R. Ardito, N. M. Pugno, and A. Corigliano, "A design strategy to match the band gap of periodic and aperiodic metamaterials," Sci. Rep. 10(1), 16403 (2020). 